\title{
Neue Beiträge zur Ikonographie von Paracelsus
}

\author{
Von J.STREBEL, Luzern
}

Karl Aberle unterscheidet in seiner Arbeit über «Grabdenkmal, Schädel und Abbildungen des Theophrastus Paracelsus» (Mitteilungen zur Salzburger Landeskunde, 1887) acht Typen von Paracelsus-Bildnissen, von denen wir mit dem letzten ausführlichen Ikonographen Professor G.F.Hartlaub (Heidelberg) die drei letzten streichen können. Es bleiben dann noch die bekannten fünf Typen: der HolLAR-Stich, das sogenannte Rubens-Bild mit der Stadt Dinant im Hintergrund, das sogenannte TinTORETto-(RoBusti-)Bild, das authentische charakteristische HirschvogelPorträt 1538 und das ebenso authentische Brustbild des gleichen Monogrammisten AH, dessen Kupferplatte von 1540 die «Albertina» in Wien besitzt. Hier sei nur der Typus I: der sogenannte Hollar-Stich, analysiert, da auch hiebei noch viele Fragen unbeantwortet sind seit den Forschungen von Aberle und Sudhoff (1922). Durch Zufall kam ich in den Besitz eines Originalstiches von Wenzel Hollar, von dem ich in meiner Stichsammlung über hundert Blätter, auch die nach NAGLER sehr seltenen $J a h$ reszeiten besitze. Ein Fräulein HeLfennberger, jetzt im Berner Oberland lebend, brachte mir vor zehn Jahren die Hand- und Denckbibel Aureoli Theophrasti Paracelsi (Franckfurt vnd Leipzig/Verlegts Andreas Luppius, Buchhändler in Nimmägen, Ann.1684) mit einem prachtvoll erhaltenen Hollar-Stich (Abb. 1). Das Helfenbergersche Exemplar soll schon seit mehr als vier Generationen in der sanktgallischen Ärztefamilie der HeLfenBerger neben sehr zahlreichen Paracelsica, auch handgeschriebenen Analecta et Excerpta, gewesen sein, welch letztere der Bruder von Frl. H. vor etwa fünfundvierzig Jahren ins Ausland verkauft habe, während Frl. H. aus der Paracelsischen Sammlung, die ursprünglich aus der Sankt-GallerKlosterfiliale Magdenau stammte, von wo sie an die Doctores HelfenBERGER gelangte, eben diese Denkbibel gerettet habe. Noch 1921 war in Luzern eine Originaldruckschrift der seltenen Nürnberger Erstdrucke über die Syphilis (Peypus-Druck 1530) erwerbbar.

Was berechtigt uns, diesen fein ausgeführten Kupferstich dem WenzeL Hollar zuzuschreiben? Signiert ist er nicht. Hollar hatte eine Schwäche, Muffe und Pelze darzustellen. Auch Paracelsus hatte in seiner Jugend eine Schwäche für Marderpelze, wie aus dem Inventar nach dem übereilten 
Abschied von Salzburg, 1525, hervorgeht, was SudHoff hervorhebt. HoLLAR hat in London seine Frau, die ihm beim Radieren half, angelernt, Pelze und Muffe nach seiner Manier minutiös zu sticheln. So hinterließ er selten gewordene Blätter mit feinstgestichelten Muffen und Pelzsorten. Auch beim vorliegenden Porträt erkennt man sofort die Hand Hollars an der rembrandtesk-helldunkel gestichelten Pelzschaube, worauf noch nie aufmerksam gemacht wurde. Aberle führt andere, gewichtigere Gründe für die Autorschaft Hollars an. Er verglich in der «Albertina», die ihm alle Stiche von Hollar und Rembrandt zur Verfügung stellte, Manier und Schrift und fand sie völlig übereinstimmend, z.B. mit dem echten Hollar-Porträt des Joanes Princeus Britanus 1644. Weitere Argumente für die Autorschaft von Hollar erläutert Aberle ausführlicher in der Fortsetzung seiner Arbeit 1888, S.272 ff. l.c. AberLe fand nämlich in der «Albertina» einen Plattenabdruck, der identisch ist mit unserem, auf dem ausdrücklich Wenzel Hollar als Stecher angegeben ist. Dazu kommt noch die Aberle von Custos Dr. Karpf mitgeteilte Notiz, daß im 1.Bande des Catalogue raisoné du cabinet d'estampes de feu Mr. Winckler, Banquier et Membre du Senat à Leipzig (1802) unter Nr. 2182 unser Stichblatt verzeichnet ist mit Signatur «Wenceslas Hollar. Aureolus Philippus Theophrastus Paracelsus, Bombast ab Hohenheim, buste in 12». Also wurde noch zu Anfang des 19. Jahrhunderts Hollar als sicherer Verfertiger dieses geätzten Bildes gehalten. Vermutlich gelangte dieser Titelkupfer aus der Leipziger Sammlung in die «Albertina». Die oben erwähnte Denkbibel ist allerdings erst 1684, also sieben Jahre nach dem Tode Hollars gedruckt worden. Wahrscheinlich, schreibt Aberle l.c. S.276, 1884, hatte der Buchhändler Luppius in Nimwegen die Platte schon früher bestellt oder aus dem Nachla $\beta$ von Hollar erworben. Ganz in die Irre geht nun Aberle, wenn er das Original oder Vorbild von Rembrandt oder einem seiner Schüler gemalt vermutet.

Woher hatte Hollar sein Vorbild? Daß das Blatt von Hollars Hand stammt, ist unzweifelhaft. Sogar der ungeübte Kunstkenner AberLE schreibt: «Seine Manier ist so charakteristisch, daß die Abstammung nie zweifelhaft war.» Ohne weiteres finden wir das Vorbild ohne die diesbezüglichen Studien Sudhoffs, die mir im Original noch nicht zugängig waren, in der Biographie von Wenzel Hollar selbst, der als Adeliger von Prachna 1607 in Prag geboren, seiner Güter beraubt, seine Liebhaberei des Zeichnens und Kupferstechens zum Lebensunterhalt benutzen mußte. Zwanzigjährig verließ er das ungastliche Prag, begab sich auf die Wanderschaft, 
studierte schon 1627 und später erneut in Basel die HoLbein-Zeichnungen der ehemaligen Amerbach-Sammlung und lernte in Frankfurt die Finessen der Radierkunst beim Basler Kupferstecher Mathes Merian, worin er seinen Meister in der Zierlichkeit der Grabstichelführung bald weit hinter sich ließ, wie NAGLER in Band VII des Künstlerlexikons schreibt. 1636 nahm ihn in Köln Sir Thомаs Howard, Graf von Arundel, als Illustrator seiner Wanderfahrten und Kupferstecher in seine Dienste. Mit Graf ArunDEL als Gesandtem am kaiserlichen Hof in Wien reiste er von Köln nach Linz, Wien und London, wo er 1677 in Armut starb. Auf der Rückreise berührte die Reisegesellschaft u.a. Luzern, Basel, Straßburg, Köln, Antwerpen, Mecheln usw., wobei Hollar den Auftrag hatte, die Kupferstichkabinette und Zeichnungssammlungen zu besichtigen und passende Stücke zu kopieren. Zweimal konnte Hollar so in Basel die Holbein-Zeichnungen studieren und kopieren, auch die feine, durchgeistigte Zeichnung von HoLBEIN : Junger Mann mit Barett oder richtiger mit Schlapphut, das wohl kurz vor der Abreise HoLBeins nach England, 1526, entstand, wohl im Auftrag des Freundes von Paracelsus, des Kunstsammlers Dr.jur. Bonifacius Amerbach. Wenn nun Hollar mit apodiktischer Sicherheit unter seinen Stich, hundertundein Jahre nach HoLBeINs kolorierter Kreidezeichnung, die genau den Jungen Mann mit Schlapphut des jüngeren Holbein darstellt, ätzen durfte: Aureolus Philippus Theophrastus Paracelsus, Bombast ab Hohenheim, Trismegistus Germanicus, so mußte in Basel eine ganz sichere Tradition sein von den Amerbach-Sammlern her, daß der «Junge Mann mit Barett oder Schlapphut», wie er kurzerhand später von der Kunstgeschichte bezeichnet wurde, niemand anders als Paracelsus war, der sich am 5. Dezember 1526 das Straßburger Bürgerrecht gekauft und schon früher von Straßburg aus öfter mit den Basler Humanisten verkehrt hatte, wo Holbein heimisch war: bei Froben, späterem Patienten von ParaGELSUS, bei dem er sicherlich auch drucken lassen wollte, was sein jäher Tod verhinderte, sowie Paracelsi Flucht aus Basel Ende Februar 1528, ferner bei den Amerbach, die in mehreren Generationen Holbein-Kunstgut betreuten, das heute noch den Grundstock der Basler Kunstsammlung bildet, nachdem von einem späteren Erben ein Teilstück dieser Sammlung nach Deutschland verkauft wurde und heute die Dessauer Sammlung ${ }^{1}$ bildet, wie ich erstmalig in Heft 6, Serie II, der «Bibliophilen», 1936, nachgewiesen habe. Mittelpunkt dieses Humanistenzirkels war bekanntlich Erasmus

${ }^{1}$ Durch den zweiten Weltkrieg größtenteils vernichtet. 
von Rotterdam, der Paracelsus sein Consilium in einem noch erhaltenen Brief verdankte.

Der Hollar-Stich der Hand- und Denkbibel ist nach übereinstimmendem Urteil aller Kunstsachverständigen nichts anderes als eine Kopie im Gegenstich des Holbeinschen Jungen Mannes mit Schlapphut, das Professor H.A. Sснмп in den Schaubüchern 8 mit der Jahrzahl 1528 datiert, was nicht stimmt. Glied um Glied habe ich die Beweise neu zusammengefügt, daß der Junge Mann mit Schlapphut des Basler HoLbein-Kabinettes niemand anders sein kann als Paracelsus. Reproduziert ist diese farbige Kreidezeichnung in der Monographie Hans Holbein der Jüngere von PAUL GANz S. XXXI als Bildnis eines Unbekannten. In diesem Werk finden sich S. 259 nicht weniger als vierzehn Stichkopien von WENzEL HoLLAR, der auch in England entstandene Werke Holbeins kopierte. Holbein d.J., geboren 1497 in Augsburg, hielt sich erstmalig mit seinem in früher Jugend an Tuberkulose verstorbenen Bruder Brosi 1514-17 in Basel auf. Hernach treffen wir ihn 1517-19 in Luzern (Hertensteinhaus) und Italien, wieder in Basel 1519-26, drittmalig 1528-31. Professor GANz wagt keine Datierung für das überall bekannte schöne Bildnis des Jungen Mannes mit Schlapphut oder Bildnis eines Unbekannten. Sie kann nur auf 1526 lauten, da PARAcelsus Ende Februar 1528 Basel verlassen mußte. S. 196 der Monographie von Ganz findet sich nochmals ein Stich von Hollar reproduziert als Bildnis eines Jünglings, gleichsinnig, aber sehr konventionell gestochen nach HoLBEINs Paracelsus, während der Gegenstich der Hand- und Denkbibel individualisiert ist, jedoch genau das gleiche Falbelhemd mit Pelzschaube aufweist. Farbig sehr schön reproduziert ist diese HoLberN-Zeichnung der Basler Kunstsammlung in Nr.95 der Insel-Bücherei als Nr. 5 mit der Bezeichnung als «Theophrastus von Hohenheim, genannt Paracelsus. 1526.»

Schon in meinen «Vererbungsstudien an Paracelsus» bin ich für die Identität der Holbein-Zeichnung mit Paracelsus eingetreten, was von einem namhaften schweizerischen Kunsthistoriker (L.B.) bestritten wurde. Deshalb sei der Heidelberger Kunsthistoriker Professor G.F.Hartlaub aus Nr. 10 der «Kunst-Rundschau» 1941 zitiert: Hollar hat das Blatt der Amerbach-Sammlung kopiert und einen Kupferstich im Gegensinn mit etlichen Varianten im Zeitgeschmack hergestellt und das Porträt im Sinn der alten Basler Tradition als Paracelsus bezeichnet. Hartlaub betont, daß diese «wunderbar reine HoLBEIN-Zeichnung» an Qualität das beste der erhaltenen Bildnisse sei, womit er nicht sage, daß es auch das am meisten charakteristische darstelle. Dazu war Holbeins Art zu kühl. Seine Zeich- 
nung des blondhaarigen jungen Mannes ist trotz verjüngender Idealisierung gewiß ähnlich und präzis: kennzeichnend sind die scharfe dinarische Adlernase, die kleinen kritisch musternden Augen und die überall wiederkehrenden melancholisch und skeptisch herabgezogenen Mundwinkel. Welch ein Unterschied in den Bildnissen des 33jährigen draufgängerischen Paracelsus von Holbein und dem des müden, vorzeitig gealterten, abgekämpften Forschers von Augustin Hirschvogel 1538 und 1540. Auch deswegen bestritt mir ein Kunsthistoriker die Identität der HolbeIN-Zeichnung mit dem jungen Paracelsus der Straßburger Zeit. Völlig zu Unrecht. Denn beide zeigen die dinarische Nase der Berglerköpfe und den verachtungsvoll nach unten gezogenen Mund. Auf dem Holbein-Hollar-Stich scheint P. noch nichts von seinem Blondhaar, das ihm von seiten des Vaters den Beinamen Aureolus, Blondel oder Golder eintrug (SudHoff) eingebüßt zu haben, während ihm die Hirschvogel-Porträts nur mehr das «Gauchhaar» lassen, was bei seinem ständigen Laborieren mit $\mathrm{Hg}$ - und As-Dämpfen erklärbar ist, was ihm nicht nur Haare kostete, sondern auch eine Leber- und Nierenvergiftung verursachte, an der P. im typischen Nephritisalter von achtundvierzig Jahren starb. Ein Jahr vor seinem Ableben konnte er «schweren Leibes halber» nicht mehr zu Pferde steigen infolge Lebercirrhose mit Ascites. Daher das verfallene kachektische Gesicht des von früher Jugend an Rachitischen mit stärkerer Skelettierung der Nasenpartie im HiRschvOGEL-Porträt. HoLBEIN charakterisiert treffend den draufgängerischen Idealisten, während Hirschvogel im Profilbild 1538 realistisch den bereits vom Tod gezeichneten abgekämpften Forscher darstellt.

\section{II}

In Heft 3/4, 1948, des «Gesnerus» wird in der Arbeit «Neues vom St. Galler Schobinger-Bildnis des Paracelsus» dargetan, daß die Schobinger für ihre Familienangehörigen Porträtisten beauftragten, die auf Holztafeln malten, wie noch vorhandene ScновіNGer-Porträts der 16. Jahrhunderts beweisen, daß ferner BARtholomäus Schobinger, der «Reiche», ein Bildnis des bärtigen Hohenheimers hat malen lassen. Von diesem steht fest, daß es verschollen ist und daß das heute in der Apotheke des Historischen Museums deponierte Ölbildnis mit dunklem Vollbart und der falschen Datierung «Theofrastus Parracelsus 1529» aus dem 17.Jahrhundert stammt und nicht das Original ist, sondern eine wahrscheinlich während der ScноBINGERschen Erbstreitigkeiten 1652-57 untergeschobene Kopie desselben. 
Dafür spricht die im Basler Kunstmuseum auf meine Veranlassung gemachte Röntgendurchleuchtung, die das Bild als eine Kopie des 17.Jahrhunderts nachwies, nicht etwa als übermaltes Originalbild. Dafür spricht auch die Eintragung eines Schовinger in den Bibliothekskatalog um 1657, der um den Sachverhalt wußte: ex archetypo depictus, was Kopie bedeutet.

Sudhoff bemerkt in seinem Paracelsus-Lebensbild 1936 dazu: Dieses Paracelsus-Bildnis war lange als geschätzter Familienbesitz in St. Gallen bei den SchoвINGER, ist ihnen aber später verloren gegangen und war lange verschollen. Was in der Mitte des 19. Jahrhunderts wieder auftauchte, halte ich für eine plumpe Unterschiebung. Schon die Jahrzahl 1529 zur Rechten des Kopfes ist für St. Gallen völlig unmöglich, ebenso für den blonden Hohenheim der üppige schwarze Bart. Bärtig soll das Bild gewesen sein, also wie das Tintoretro-Bild (auf dem Chauvéau-Stich der Genfer De-TournesAusgabe 1658). Es wäre sehr zu wünschen, daß das wirkliche Schobingersche Paracelsus-Bildnis wieder auftauchte.» An diesen Wunsch Sudhoffs dachte ich, als ich vor etwa einem Jahr aus Amerika Kenntnis bekam, daß dort ein bärtiges Paragelsus-Bildnis vom St.Galler oder TintorettoTypus aufgetaucht sei. Da mir schon zwei offenkundige Fälschungen des Rubens-Typus in zwei bekannten Sammlungen zu Gesicht gekommen waren, mußte ich sehr skeptisch sein. Aber selbst sehr schlechte Photos zeigten mir ein selten beseeltes Paracelsus-Gesicht mit Bart, das in der Beschriftung, Malerei, Feinbehandlung der Hände und des Schwertknaufs nur von einem italienischen Meister stammen konnte. Alles wies auf Trntoretto-Robusti, dessen Paragelsus-Bildnis weder Aberle (1888), SudHOFF (1922) oder HaRTlaub (1941) zeitlich einordnen konnten, obwohl dies nicht schwierig ist. Es dürfte 1535 im Veltlin entstanden sein, als Paracelsus verwahrlost von Innsbruck über die damals reiche Handelsstadt Sterzing in schäbiger Schaube übers Penser Joch lief, weil er kein Geld weder für Pferde noch für einen neuen Mantel hatte. Roвusti, genannt das Färberlein (Tintoretto), hat ihn in dieser schäbigen Schaube als «Santo» dargestellt. Er gleicht darin, auch im verwilderten Bart, auffällig dem sogenannten «Läuseheiligen» von Labre, von dem ich ein Originalbildnis besitze. Sogar eine leichte Aura umstrahlt den abgezehrten Bärtigen, der von sich schrieb: «Mein Bart hat mehr erfahren als der Schulsack eurer Hohen Schulen, die noch niedrig genug werden.» Bitiskius gibt in seiner Vorrede zur Genfer De-Tournes-Ausgabe 1658 (die nach eigenen Untersuchungen ein lateinisches Plagiat der Palthenius-Edition darstellt) an, daß der Vorsatz-Stich von Chauvéau, der den ersten der drei Bände ziert, nach dem 
Original von JACOPo RoBUsti (Tintoretto) sei, der «ad vivum pinxit» und zwar «juniore Theophrasto Venetorum Martem sequente», was nicht stimmen kann, da die Kämpfe der Venetianer gegen die Kaiserlichen 1521-25 fallen, während RoBusti 1512 in Venedig geboren ist. Unsere Datierung von 1535 dürfte der Wahrheit entsprechen, die auch Fridericus Bitrscius 1658 nicht mehr bekannt war. Auch Aberle entging die Notiz des BrTscIUs. Leider konnte ich das Original, das sich zurzeit in New York befindet, nicht einsehen, wohl aber Röntgenbilder, aus denen hervorgeht, wie auch aus den Photos, daß es sich um ein Bildnis des 17. Jahrhunderts handelt. Damit scheiden der Sankt Galler Porträtist wie TrNoRETto wiederum aus. Aber irgendwann muß Tintoretto hier Vorbild gewesen sein. So lautet nicht nur meine unmaßgebliche Meinung, sondern die von namhaften Kunstsachverständigen. Auch dieser Fragenkomplex sollte weiter untersucht werden von der zurzeit kompetentesten Beurteilerin, Frau Dr.Margarete Pfister-Burkhalter, erste Assistentin am Kupferstichkabinett des Kunstmuseums Basel, die bereits einen Zettelkatalog über die Paracelsus-Bildnisse angelegt hat. Das Sankt Galler Bildnis ordnete sie in die Reihe der Bilder in Schleißheim und Salzburg, die auf ein italienisches Original zurückgehen, wofür u.a. das $f$ statt $p h$ in «Theophrastus» spricht. Auch bei diesem neu aufgetauchten, unbeschriebenen, vorzüglich gemalten Bildinis Paracelsi des 17.Jahrhunderts steht Theofr. mit $f$ und Azot ohne h. Neben der Holbmin-Zeichnung 1526 ist dieses neue Bildnis Hohenheims das seelenvollste, das mir je zu Gesicht gekommen ist.

\section{III}

Als Titelblatt des IV. Bandes der Zollikoferschen Paracelsus-Ausgabe ist eine Federzeichnung reproduziert, die den Hohenheimer nach dem Rosenkreuzerporträt des dritten kölnischen Byrckmann-Bandes in weiterentwickelter adeptischer Symbolbegleitung darstellt. Da ich mich in drei Arbeiten: «Paracelsus und die Rosenkreuzer» (III. Bd. der Nova Acta Paracelsica), «Über die Byrckmann-Drucke 1564/67», «Über Entstehung und Bildkomponenten des sogenannten Rosenkreuzerporträts Hohenheims im kölnischen Byrckmann-Holzschnitt von Fravz Hoogenbergh 1567» (IV. Bd. der NAP), zu diesem interessanten Porträt der Kölner Adeptenschule des 16. Jahrhunderts geäußert habe, möchte ich dieses neugefundene Porträt PARacelsi nicht nur hier einreihen, sondern es kurz besprechen, da es im IV. Band der Paracelsus-Ausgabe nicht weiter analysiert ist. Die Re- 


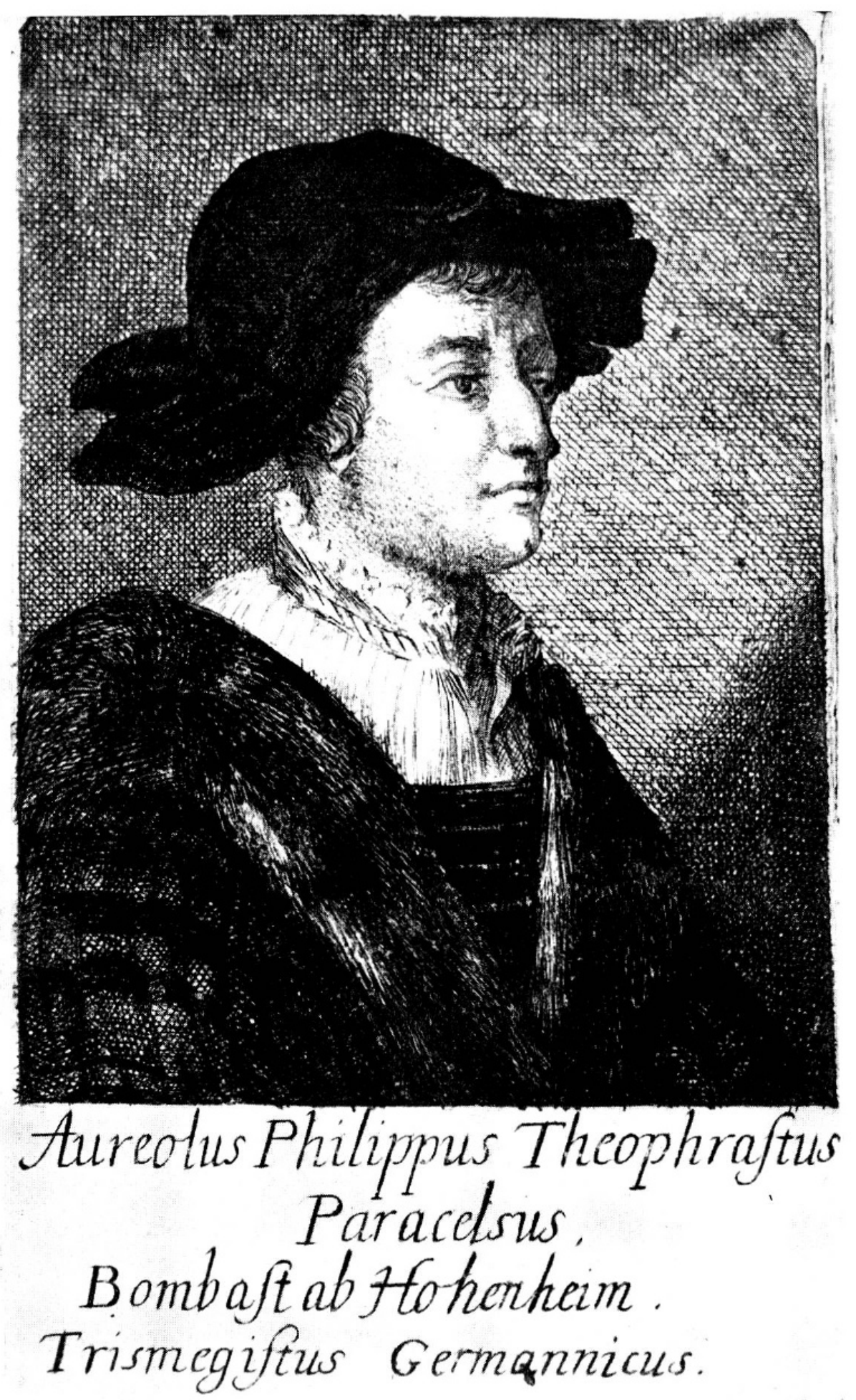

Hollar-Stich 1627 aus der Paracelsischen «Hand- vnd Denckbibel» nach der HoLbein-Zeichnung 1526 der AMERBAch-Sammlung in Basel (Facsimile der Original-Radierung) 
produktion ist dort zu vergleichen. Die Federzeichnung ist auf Nürnberger Papier mit dem Wasserzeichen der sogenannten hohen Krone feingestrichelt mit schwarzer Tusche. Das Papier weist auf das 16. Jahrhundert. DüRERsche Probedrucke weisen häufig das nämliche Wasserzeichen auf. Sein Urbild ist die Radierung des Monogrammisten AH anni 1540 oder vielmehr, da es ein Spiegelbild dieses Stiches darstellt wie der Byrckmannsche Holzschnitt von Franz Hoogenbergh, die zeichnerische Kopie des HoogenBERGHschen Holzschnittes. Nicht nur das Bild im Gegenstich spricht dafür, sondern die Mauergliederung im Hintergrund mit den herabhängenden Moosfetzen (genau wie im Titelblatt der Erstausgabe des Labyrinthus medicorum errantium: Anno MDLIII, reproduziert S. 140 des I.Bandes der Zollikofer-Edition), wie auch der Ausblick in die Landschaft links oben sowie der ganze Habitus des Dargestellten. Falbelhemd mit Halskrause, Wappenrock mit Amulettskapulier, Adelsschwert mit Großknauf, auf dem das Azoth prangt als Symbol der höheren und niederen Alchimie, finden sich genau wie bei den Originalstichen Hirschvogels. Wenn man die sehr schlecht gezeichneten Arme, die wurstförmig und links wie ausgerenkt dargestellt sind, mit den krassen Extremitätenverzeichnungen HIRSGHVogelS in der Bilderserie seines Biographen Scнмidт vergleicht, so könnten gewiegteste Kunstkenner darauf kommen, zu behaupten, daß wir hier eine Originalzeichnung von Hirschvogel selbst vor uns hätten. Dafür würde nicht nur die für Hirschvogel typische Verzeichnung der Extremitäten«Flossen» sprechen, analog seiner Kleopatra-Verzeichnung und sieben andern, sondern auch das Versteckspiel der Hände, die Hirschvogel stets unproportioniert klein verzeichnet hat, weswegen er sie mit Vorliebe ungezeichnet ließ, sowie das Nürnberger Wasserzeichen des 16. Jahrhunderts. HirschVogel stammte aus Nürnberg und begab sich von Klagenfurt, wo er Paracelsus traf, und Wien gelegentlich wieder an seinen Geburtsort. Hirschyogel war auch Geometer, hat ein Lehrbuch der Geometrie verfaßt analog Dürer, der eines über Perspektive usw. schrieb. Vielen seiner Zeichnungen gab er deshalb das Richtmaß des Dreiecks bei, wie es rechts oben gezeichnet ist anstelle der Adeptensymbole im Byrckmannschen Holzschnitt. Stutzig macht hingegen die Symbolgestaltung im linken oberen Quadranten mit der aufgehenden Sonne, welche die logische Weiterentwicklung der Adepten und ursprünglich rein mystisch orientierten Rosenkreuzer zu gewissen Logen dartut. Schon Pastor Johannes Valentin Andreae, Verfasser der Fama Fraternitatis der Confessio Rosicruci und der Chymischen Hochzeit des Christian Rosenkreuz, strebte eine solche Entwick- 
lung an, die der Dreißigjährige Krieg vernichtete. Vorläufer war schon der Kusaner, der von seinem Basler Konziliumsfreund Aeneas Silvio PiccoLOMINI, dem späteren Papst Pius II, nicht nur auf die Suchenach griechischen Manuskripten nach Konstantinopel geschickt worden war, sondern in der Hauptmission zur Unierung der oströmischen Kirche im Geist der heutigen Ökumene. Nachfahren waren Leibniz und Friedrich von Hardenberg (Novalis), welch letzterer in seinem visionären Essay Die Christenheit oder Europa in edler Sorge um die heute erfolgte Zersplitterung der Menschheit durch das Medium des geeinten Christentums ein geeintes Europa erträumte, was der «alte Heide» GoEthe als Befürworter der Antike als Rückfall ins Mittelalter geißelte. Immer wieder wurden Fraternitäten gegründet analog unserem ehemaligen Völkerbund. Immer wieder suchte man Leitideen und «Führer». Da geistesmächtige Köpfe wie der Kusaner, Leibniz und NovaLIS erkannten, daß politische Normen allein niemals zu einer Einigung führen können, weil Politik letzten Endes materiell, nicht spirituell orientiert ist und auseinanderstrebende Interessengruppen vertritt, strebten sie nach einer Einigung im Sinne des Tatchristentums der Pfingstgemeinde. Vorbilder waren den Rosenkreuzern des 17. Jahrhunderts der Kusaner und vor allem - Paracelsus, obwohl er «nit in vnsere Fraternitet getretten», wie Andreae schreibt. Dieser Satz beweist, daß schon zu Lebzeiten Paracelsi Rosenkreuzer-Alchimisten existierten, deren Adepten Hoнenнeim selbst «einen weiten Weg nachgereiset», wie er selbst im Vorwort mit Widmung an den Adepten Dr. jur. Brant, Pfarrer in Eferding, in seiner letzten Fassung der tartarischen Krankheiten schreibt. Sogar im sagenhaften Grabe des Christian Rosenkreuz, also am heiligsten Ort dieser Mystikergesellschaft, fanden sich laut AndreaE «alle vnsere Bücher sampt deme Vocabulario Theoph: P. ab Ho», also ein Schriftwerk Hohenheims. Findet sich das am heiligsten Ort, dann steht die Schrift und ihr Verfasser im hohen Wert. Von hier aus über Noluius, Weigel, Andreae, Böhme, Goethe führen vielfach verschlungene Pfade zu den heutigen Rosenkreuzern, die alle ihrem Ahnherrn, dem Hohenheimer, ihre Referenz mit magisch-mystischen Zutaten erwiesen, wie auch aus der in unserem IV. Paracelsus-Band reproduzierten neugefundenen Zeichnung wahrscheinlich des 16.Jahrhunderts hervorgeht. Ohne Kenntnisnahme der Rosenkreuzer-Symbole lassen sich diese Bildnisse überhaupt nicht richtig analysieren. Hochinteressant sind diesbezüglich die Bildkomponenten des sogenannten RosenkreuzerPorträts Hohenheims des kölnischen Byrckmann-Holzschnittes von Franz Hoogenbergh 1567, das im IV. Jahrbuch der NAP, S.122, erstmalig ge- 
nau analysiert ist. Dr. med. Theodor ByrckmanN in Köln erhielt durch Vermittlung der Rosenkreuzer in Wien etwa fünfundzwanzig Jahre nach Paracelsi Tod einen Abzug des Hirschvogel-Porträts 1540 zusammen mit dem sogenannten «Kärntner Trio »(1538) der Defensiones, des Labyrinthus, der letzten Tartarusarbeit, und der Chronik des Landes Kärnten. Ohne die Bemühungen der Kölner Rosenkreuzer wäre dieses Trio, das unter den Standesakten von Klagenfurt vergraben und vergessen war, verloren gegangen. Vom Holzschneider Franz Hoogenbergh ließ Dr. med. Th. Byrckmann nach Erhalt des Trios mit Plattenabzug in der väterlichen Druckerei Byrckmannis Erben in beiden oberen Bildquadranten rosenkreuzerische Adeptenzeichnungen anbringen: links die Geburt zum irdischen Licht, signiert mit Kreuz und Rosa, rechts die symbolisch sehr interessant dargestellte Geburt des vorgeschrittenen Adepten zum himmlischen Licht (die zwei «Lichter» Hohenheims). Dieses unzweideutige Signet beweist, daß die Rosenkreuzer schon fast hundert Jahre vor dem vorgeschützten Christian Rosenkreuz existiert haben, deren Wirken durch die Druckoffizin Byrckmann in Köln über die von Lechler-Feyerabend in Frankfurt am Main, die alte «Pfaffenstraße» des Rheins über Straßburg und die Wasserstraße der Donau (Neuburg mit den Paracelsischen Manuskriptensammlungen) nach Wien und Schlesien (Montanus) reichte und u.a. das Kärntner Trio gerettet hat. Die Ergebnisse meiner früheren Arbeit über «PARAcELSUS und die Rosenkreuzer» im III. Band der NAP sind heute allgemein anerkannt, so daß Francis Kordas, F. R.C., in mehreren Artikeln im «Rosicrucian Digest» 1950 über «PARAGelsus the Rosicrucian» schreiben konnte: Few people know that Paracelsus was not only one of the greatest mystics of the Middle Ages, but also an initiated Rosicrucian.»

\title{
Ein unbekannter Brief von Johann Heinrich Lambert an Johannes Gessner
}

\author{
Von Max StecK, zurzeit Basel
}

1. Vor genau hundert Jahren hat der durch seine astronomischen Forschungen wie durch seine Biographien zur Kulturgeschichte der Schweiz gleich bedeutende Forscher RudolF Wolf in der berühmten Briefsamm- 